\title{
Nutrizione parenterale intradialitica: indicazioni, aspetti pratici e limiti
}

\author{
Alice Sabatino, Emanuele Trenti, Enrico Fiaccadori
}

Unità di Fisiopatologia dell'Insufficienza Renale Acuta e Cronica, Dipartimento di Medicina Clinica e Sperimentale, Università degli Studi di Parma, Parma

Intradialytic Parenteral Nutrition: practical aspects and management in End-Stage Renal Disease

Abstract. Protein-Energy Wasting (PEW) is common among patients with End Stage Renal Disease (ESRD) and is associated with a significant increase of the already high morbidity/mortality risk in this population.

Since one of the main mechanisms of PEW in ESRD is the insufficient nutrient intake, oral nutritional supplements have been extensively used in this clinical setting, and they have been demonstrated to be very efficient in preventing and treating PEW. Also, other approaches have been proposed as nutritional support modalities, such for example intradialitic parenteral nutrition (IDPN). However, despite the demonstration of nutritional and metabolic status improvement by IDPN, there is still poor evidence relating this modality with reduction in hospitalization rate and mortality risk.

On this basis, the present review is aimed at reviewing nutritional support practice in the treatment of PEW in ESRD, with a more in depth focus on the role of IDPN as a modality of nutritional supplementation. To this purpose, indications and quantitative/qualitative aspects of IDPN, as well as IDPN management, safety issues and limitations will be discussed.

Key words: Intradialytic parenteral nutrition, End stage renal disease, Protein-energy wasting, Nutritional supplementation

Conflict of interest: None.

Financial support: None.

Accettato: 29 Maggio 2014

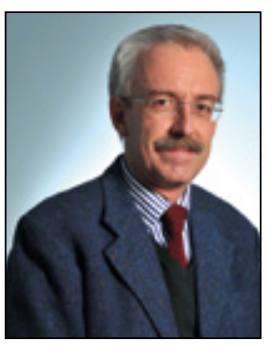

Enrico Fiaccadori

\section{Introduzione}

In corso di malattia renale cronica (Chronic Kidney Disease, CKD) la deplezione proteico-energetica (Protein-Energy Wasting, PEW) si caratterizza per la progressiva riduzione del patrimonio proteico ed energetico dell'individuo (1-3).

La prevalenza della PEW è particolarmente elevata nella CKD e aumenta in rapporto al peggioramento della funzione renale: in numerose casistiche è stata riscontrata una condizione di PEW nel 28-48\% dei pazienti nei primi 4 stadi della CKD e fino al 50-75\% dei soggetti in dialisi cronica (End Stage Renal Disease, ESRD) $(1,4,5)$.

La PEW è uno degli fattori di morbilità più rilevanti nel paziente nefropatico e si configura come un fattore altamente predittivo di mortalità (6).

Numerosi fattori patogenetici, attraverso meccanismi differenti, sono in grado di esercitare un impatto negativo sullo stato nutrizionale dei pazienti con CKD/ESRD, portando progressivamente allo sviluppo della PEW e ai suoi molteplici effetti negativi $(2,7)$. In tale contesto clinico, una delle cause principali della PEW è rappresentata dall'assunzione inadeguata di nutrienti, generalmente combinata ad altri fattori di rischio connessi alla CKD, al suo trattamento ed alle complicanze/alterazioni metaboliche ad essa correlate (anemia, deficit di vitamina $\mathrm{D}$, acidosi metabolica, stress ossidativo, infiammazione, ecc.) $(2,8)$. Negli stessi pazienti, anche età avanzata, fragilità/povertà e numerose comorbilità acute/ croniche (p. es., diabete, sindrome metabolica, insufficienza cardiaca, sovraccarico di liquidi, epatopatie e infezioni) possono concorrere in misura rilevante a ridurre l'apporto di nutrienti $(2,3)$.

I principali fattori che si ritiene svolgano un ruolo importante nel ridurre l'assunzione di nutrienti nella $\mathrm{CKD} / \mathrm{ESRD}$ sono l'anoressia (perdita di appetito) e le restrizioni dietetiche inappropriate (2-4). La perdita progressiva dell'appetito è stata documentata in una percentuale elevata di soggetti con CKD $(35-70 \%)$ ed è presente già negli stadi più precoci $(2,9)$; la perdita di appetito è legata a una complessa interazione negativa tra segnali metabolici, accumulo di tossine uremiche, deficit nei normali meccanismi omeostatici del sistema digestivo, alterazione dei livelli circolanti dei regolatori dell'appetito (mediatori gastrici, adipochine e citochine) e alterato signaling ipotalamico $(2,9)$. Inoltre, nei pazienti con CKD in 
trattamento conservativo, è frequente una storia di restrizione dietetica di lunga durata per vari nutrienti; in questi pazienti, nonostante l'assunzione controllata di proteine, fosforo, potassio e sodio sia in grado di prevenire importanti complicanze della $\mathrm{CKD}$, oltre a rallentare la progressione della malattia renale cronica (10), un'eccessiva riduzione dell'assunzione di calorie e proteine può determinare importanti effetti negativi sullo stato nutrizionale (11).

Su queste basi fisiopatologiche, negli ultimi anni, particolare attenzione è stata rivolta alla supplementazione nutrizionale per via orale durante la dialisi e/o nell'intervallo interdialitico, associata ad un accurato counselling dietetico. Oltre a essere un approccio semplice e relativamente a basso costo, la supplementazione nutrizionale per via orale si è dimostrata efficace nel prevenire e nel trattare la PEW (12-14).

La nutrizione parenterale intradialitica (IDPN), per esempio la somministrazione di nutrienti direttamente nel circuito della dialisi, è un'altra modalità di integrazione nutrizionale proposta per i pazienti con ESRD (15). Attualmente, i dati disponibili riguardo ai possibili effetti dell'IDPN su mortalità e tasso di ospedalizzazione sono limitati. In realtà, nonostante esistano evidenze convincenti che l'IDPN possa migliorare $\mathrm{i}$ parametri metabolici, il bilancio azotato e lo stato nutrizionale dei pazienti con ESRD, le evidenze che correlano questa modalità di supporto nutrizionale a una riduzione del tasso di ospedalizzazione e della mortalità sono ancora scarse $(12,16)$. Questa rassegna si propone di fare il punto sulle pratiche nutrizionali più comunemente utilizzate nel trattamento della PEW in ESRD, discutendo il possibile ruolo dell'IDPN come metodica di integrazione nutrizionale per i pazienti in emodialisi. A tale scopo viene presentata una discussione approfondita su aspetti pratici, composizione, gestione, indicazione e limitazioni dell'IDPN, basata sulle evidenze disponibili nella letteratura.

\section{Consulenza dietetica, integrazione orale e nutrizione enterale}

In base al fabbisogno proteico raccomandato in dialisi (almeno $1.1 \mathrm{~g} / \mathrm{Kg} /$ die) e all'osservazione che il consumo medio osservato di proteine in tali pazienti è in realtà spesso inferiore a $0.8-1.0 \mathrm{~g} /$ $\mathrm{Kg}$ /die, una supplementazione di proteine pari a circa $0.2-0.4 \mathrm{~g}$ / $\mathrm{Kg} /$ die è frequentemente necessaria (17). Inoltre, può verificarsi in parallelo anche un deficit di calorie di entità percentualmente sovrapponibile (17). Quando il paziente è a rischio di sviluppare un deficit nutrizionale, per riduzione dell'introduzione spontanea di alimenti al di sotto di $30 \mathrm{Kcal} / \mathrm{Kg} /$ die e $/ \mathrm{o} 1.1 \mathrm{~g}$ di proteine/ $\mathrm{Kg} /$ die, si rende necessario intervenire per prevenire lo sviluppo di PEW (12). In questo caso, soprattutto in assenza di uno stato di malnutrizione conclamata, la consulenza dietetica può aiutare il paziente ad aumentare l'introduzione di calorie e proteine con la dieta, valutando le abitudini alimentari e identificando $i$ motivi e i comportamenti collegati alla riduzione dell'apporto. Nei casi in cui la consulenza dietetica non sia sufficiente per raggiungere i target nutrizionali con l'alimentazione spontanea, si raccomanda l'associazione di un supplemento orale (18-20). Ciò ha portato in molti paesi d'Europa alla distribuzione di pasti o alla somministrazione di supplementi industrializzati per via orale anche durante la dialisi. Dal momento che i supplementi presenti in commercio hanno una densità calorica e proteica elevata, tra 1.8 e $2.0 \mathrm{Kcal} / \mathrm{mL}$ e un contenuto proteico di circa 70-85 g di proteine/L, la somministrazione intradialitica di una confezione (di solito circa $200 \mathrm{~mL}$ ) è in grado di garantire un introito settimanale di 1100-1200 Kcal e 42-48 g di proteine in più, mentre una somministrazione giornaliera consentirebbe un introito calorico aggiuntivo settimanale di 2500-2800 Kcal e proteico di 98-112 g (Tab. I). Le evidenze che consigliano l'uso di supplementi orali sono molteplici e dimostrano come l'utilizzazione della supplementazione orale giornaliera (14, 21) e/o intradialitica $(13,22-26)$ aumenti le concentrazioni di albumina sierica $(13,14,21-26)$ e riduca il rischio di mortalità (14) e il tasso di ospedalizzazione (13). Inoltre, studi metabolici più complessi hanno mostrato come l'introduzione di una quota di proteine per via orale durante la dialisi possa ridurre o addirittura antagonizzare l'effetto catabolico indotto dal trattamento stesso, con un effetto positivo che si protrae anche nelle ore successive alla fine della dialisi (23).

La nutrizione enterale può essere utilizzata sia come supplemento sia come nutrizione completa, nei casi di ipoalbuminemia severa $(<3.0 \mathrm{~g} / \mathrm{dL})$ refrattaria al trattamento nutrizionale e farmacologico, nei pazienti gravemente malnutriti od ospedalizzati e in casi selezionati, qualora la supplementazione nutrizionale per via orale non sia sufficiente e nei casi in cui il paziente presenti difficoltà di deglutizione. Inoltre, in alcune condizioni selezionate, la nutrizione enterale notturna attraverso una gastrostomia percutanea si è dimostrata efficace (27).

TABELLA I - QUANTITÀ DI NUTRIENTI (KCAL E PROTEINE) NEI PRODOTTI COMMERCIALI PER SUPPLEMENTAZIONE ORALE E PARENTERALE E CONTRIBUTO DELL'IDPN ALL'INTAKE CALORICO E PROTEICO COMPLESSIVO

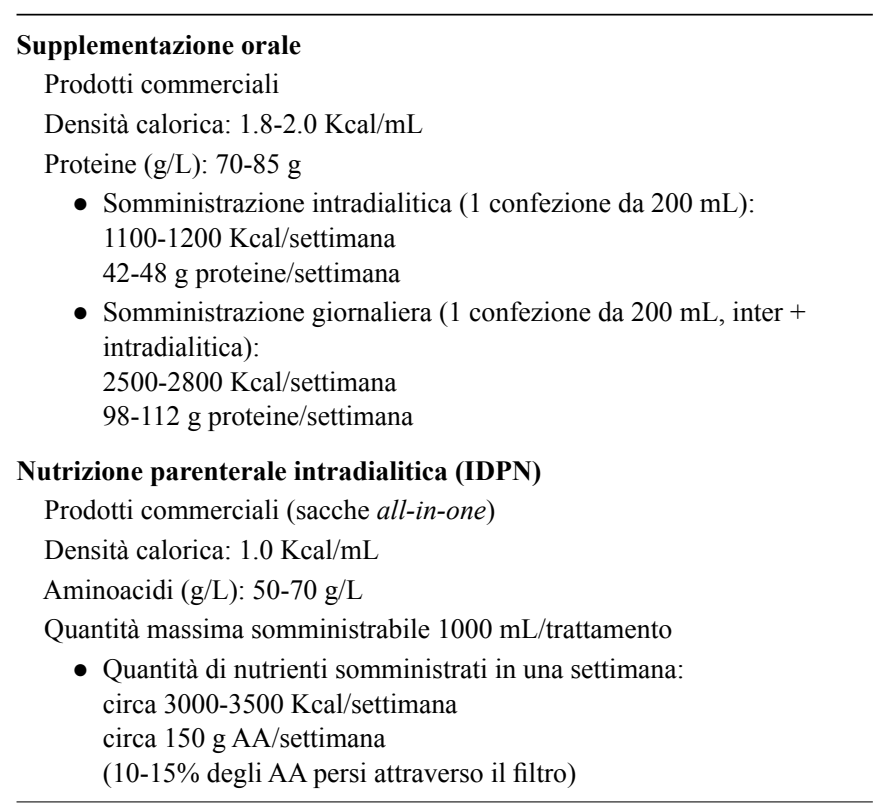

AA: aminoacidi. 


\section{Nutrizione parenterale intradialitica (IDPN)}

La nutrizione parenterale intradialitica (IDPN), cioè la somministrazione di nutrienti direttamente attraverso il circuito della dialisi, è un'altra modalità di integrazione nutrizionale attuabile nei pazienti con ESRD (15). Se si escludono le modalità non fisiologiche di somministrazione di nutrienti (p. es., glucosio, lipidi e aminoacidi infusi sequenzialmente nel circuito), esistono principalmente due modalità possibili di IDPN, che si basano rispettivamente sull'uso di sacche all-in-one confezionate dalla farmacia ospedaliera e di sacche all-in-one del commercio. Nel primo caso, nonostante il vantaggio di poter individualizzare la composizione dell'IDPN sulla base della necessità del paziente, la preparazione delle sacche presso la farmacia ospedaliera può essere molto più costosa $\mathrm{e} i$ problemi logistici possono essere ancora più complessi (trasporto sicuro e stoccaggio, breve intervallo di tempo disponibile tra la confezione e la somministrazione, ecc.). Nel secondo caso, con le sacche all-in-one del commercio attualmente disponibili, è possibile coprire le esigenze di quasi tutti i pazienti con ESRD. Inoltre, le sacche commerciali possono essere stoccate in modo sicuro a temperatura ambiente nei centri dialisi per periodi anche molto lunghi (fino a due anni) fino alla somministrazione. La composizione di alcune sacche all-in-one per l'IDPN disponibili sul mercato è descritta nella Tabella II. Generalmente sono utilizzate le soluzioni più concentrate, a causa dei tempi ridotti e della necessità di restrizione dei fluidi durante la sessione di emodialisi. In alcuni pazienti è possibile utilizzare anche soluzioni senza elettroliti (p. es., senza sodio, potassio e fosforo).

\section{Cosa ci si può aspettare dall'IDPN in termini di apporto nutrizionale?}

Per definizione, l'IDPN rappresenta una modalità di supplementazione nutrizionale con limiti di tempo importanti legati alla durata e ai ritmi della dialisi. Di conseguenza, la capacità dell'IDPN di integrare l'assunzione spontanea di nutrienti dei pazienti con ESRD garantendo il raggiungimento degli obiettivi nutrizionali dipende dalla differenza esistente tra target nutrizionale e assunzione alimentare spontanea del paziente. La densità calorica massima delle soluzioni all-in-one di solito utilizzate è di circa $1 \mathrm{Kcal} / \mathrm{mL}$ e si raccomanda di non eccedere $1000 \mathrm{~mL}$ di liquidi, $1000 \mathrm{Kcal}$ e $50 \mathrm{~g}$ di aminoacidi per dialisi. Quindi, tenendo conto dei giorni interdialitici e degli aminoacidi rimossi in ogni dialisi (28), è possibile calcolare che la quantità massima di nutrienti fornita dall'IDPN non possa superare le $3000 \mathrm{Kcal}$ e i $150 \mathrm{~g}$ di aminoacidi/settimana, che corrispondono a circa $5-6 \mathrm{Kcal} / \mathrm{Kg} /$ die e $0.30 \mathrm{~g} / \mathrm{Kg} /$ die di aminoacidi per un paziente di $70 \mathrm{Kg}$ (29) (Tab. I).

\section{Quali sono gli effetti nutrizionali dell'IDPN?}

Esistono numerosi studi sugli effetti dell'IDPN nei pazienti con ESRD (29-37), che documentano un miglioramento dello stato nutrizionale (aumento di peso corporeo, indici di massa corporea, albumina e prealbumina sierica), del bilancio proteico corporeo totale, dell'appetito e della sopravvivenza (29-34). Tuttavia, la maggior parte di questi studi non è rappresentata da RCT, l'assunzione nutrizionale spontanea non era controllata

TABELLA II - SOLUZIONI $A L L-I N-O N E$ UTILIZZABILI PER L'IDPN

\begin{tabular}{|c|c|c|c|c|}
\hline & \multicolumn{2}{|c|}{ BBraun } & \multirow{3}{*}{$\begin{array}{c}\text { Baxter } \\
\text { Olimel } \\
\text { N9/N9E }\end{array}$} & \multirow{3}{*}{$\begin{array}{c}\text { Fresenius } \\
\text { SmofKabiven }\end{array}$} \\
\hline & \multicolumn{2}{|c|}{ Nutriflexlipid } & & \\
\hline & Special & Plus & & \\
\hline Volume totale (mL) & $625 / 1250 / 1875 / 2500$ & $1250 / 1875 / 2500$ & $1000 / 1500 / 2000$ & $986 / 1477 / 1970$ \\
\hline Proteine totali (g/L) & 57.6 & 38.4 & 44.3 & 50 \\
\hline Kcal totali (1 L) & 1180 & 1012 & 1140 & 1100 \\
\hline Kcal non proteiche (1 L) & 936 & 840 & 960 & 900 \\
\hline g di Azoto/L & 8 & 5.6 & 7 & 8 \\
\hline Glucosio (g/L) & 144 & 120 & 140 & 125 \\
\hline Lipidi (g/L) & 40 & 40 & 40 & 38 \\
\hline Tipo di lipidi & PUFA + MCT & PUFA + MCT & MUFA + PUFA & PUFA (omega-3) + MCT \\
\hline Osmolarità (mOsm/L) & 1545 & 1215 & 1170 & 1500 \\
\hline $\mathrm{Na}(\mathrm{mmol} / \mathrm{L})$ & 53.6 & 40 & 35 & 40 \\
\hline $\mathrm{K}(\mathrm{mmol} / \mathrm{L})$ & 37.6 & 28 & 30 & 30 \\
\hline $\mathrm{P}(\mathrm{mmol} / \mathrm{L})$ & 16 & 12 & 15 & 12 \\
\hline Disponibile senza elettroliti? & Sì & Sì & Sì (N9E) & $\begin{array}{l}\text { Sì (soltanto le sacche con } 1500 \text { e } \\
\qquad 2000 \mathrm{~mL} \text { ) }\end{array}$ \\
\hline
\end{tabular}

IDPN: nutrizione parenterale intradialitica (intradialytic parenteral nutrition); MCT: trigliceridi a catena media; MUFA: acidi grassi monoinsaturi (monounsaturated fatty acids); PUFA: acidi grassi polinsaturi (polyunsatured fatty acids). 
e l'assunzione nutrizionale totale era generalmente inadeguata. Sono tre i RCT più recenti che hanno valutato gli effetti delle soluzioni all-in-one nei pazienti con ESRD in emodialisi (35-37) (Tab. III). In questi studi, l'IDPN ha avuto una durata variabile, da 5 settimane (36) a 1 anno (37) e solo uno di questi ha avuto un periodo di follow-up (37). Tutti e tre gli studi hanno dimostrato un effettivo miglioramento dei parametri nutrizionali; in uno di questi, sono stati dimostrati anche un incremento dell'intake nutrizionale spontaneo e una riduzione della proteina $C$ reattiva sierica (35). Nello studio con casistica di maggiore numerosità (studio FINE) (37), l'utilizzo dell'IDPN combinata con la supplementazione orale giornaliera non si associava a un miglioramento della mortalità, nonostante gli effetti positivi sulle variabili nutrizionali. Un'analisi post-hoc degli stessi dati ha suggerito che, nei pazienti in cui la supplementazione intradialitica (orale o parenterale) era in grado di aumentare i livelli di prealbumina sierica di almeno $30 \mathrm{mg} / \mathrm{L}$ entro 3 mesi, la mortalità e il tasso di ospedalizzazione a 2 anni era diminuito, indipendentemente dalla presenza di uno stato infiammatorio cronico (37).

Alcuni studi che hanno valutato gli effetti metabolici a breve termine dell'IDPN, con particolare attenzione al metabolismo proteico e alla cinetica delle proteine del muscolo scheletrico, hanno documentato un miglioramento della sintesi proteica corporea totale, una diminuzione della proteolisi e un aumento della sintesi di albumina (38-40). Di conseguenza, utilizzando l'IDPN, sarebbe potenzialmente possibile ridurre gli effetti negativi dell'emodialisi sulla sintesi e sulla degradazione proteica (41), portando a un bilancio netto positivo delle proteine. Tuttavia, a differenza di quanto si è visto con la supplementazione orale (24), gli effetti anabolici presenti nel periodo intradialitico tendono a ridursi notevolmente nel periodo post-dialisi, quando i nutrienti non sono più somministrati (24). Infine è importante sottolineare che gli effetti anabolici dell'IDPN sono potenziati qualora questa sia combinata all'attività fisica durante l'emodialisi (40).

\section{Quali sono le indicazioni dell'IDPN?}

Le indicazioni per l'uso dell'IDPN non sono ben definite, principalmente perché gli effetti positivi a lungo termine di questa modalità di approccio nutrizionale e, soprattutto, la sua eventuale superiorità rispetto ad altre strategie nutrizionali non sono mai stati formalmente dimostrati in RCT. Inoltre è opinione comune che l'IDPN non rappresenti la prima scelta, ma che, anzi, debba essere iniziata esclusivamente in pazienti in cui la supplementazione orale e il counselling dietetico non abbiano consentito il raggiungimento dei target nutrizionali. Dal momento che la quantità massima di nutrienti fornita con l'IDPN corrisponde a non più del $25 \%$ dei fabbisogni giornalieri consigliati, l'IDPN è raccomandata soltanto se il paziente assume spontaneamente almeno $20 \mathrm{Kcal} / \mathrm{Kg} /$ die $(11,20)$ e $0.8-0.9 \mathrm{~g} /$ $\mathrm{Kg} /$ die di proteine (15). I criteri clinici proposti per selezionare i pazienti più adatti all'IDPN includono generalmente la presenza di segni e sintomi di una malnutrizione severa: infatti le Linee Guida proposte nel 2000 dalla National Kidney Foundation's Kidney Disease Outcomes Quality Initiative (KDOQI) (18) suggeriscono che l'IDPN può essere efficace in pazienti malnutriti che non sono in grado di incrementare l'assunzione spontanea con l'aiuto della consulenza nutrizionale e che non tollerano l'integrazione orale o enterale, ma che assumono spontaneamente una quota sufficiente di nutrienti tale da raggiungere il target nutrizionale quando combinata con l'IDPN. Tuttavia, dato il limitato apporto di nutrienti fornito dall'IDPN, la presenza di malnutrizione severa dovrebbe portare a un approccio nutrizionale più aggressivo. È probabile che l'IDPN sia più utile nei pazienti con un elevato rischio di PEW, ma non ancora gravemente malnutriti, per i quali lo sviluppo della PEW severa potrebbe essere prevenuto da un inizio precoce del programma di supporto nutrizionale. Nonostante non sia ancora stata stabilita la durata consigliata per l'uso dell'IDPN, dovrebbe essere almeno di 3-6 mesi.

\section{Quali sono i problemi e le limitazioni dell'IDPN?}

Il rapporto costo-beneficio dell'IDPN non è ben definito. Vantaggi principali dell'IDPN: a) la supplementazione nutrizionale con soluzioni all-in-one concentrate/iperosmolari è possibile senza la necessità di un accesso venoso centrale; b) la somministrazione parenterale di nutrienti non è influenzata da problemi legati alla compliance del paziente; c) l'IDPN non è influenzata dalla presenza di eventuali alterazioni della funzione gastrointestinale; d) la quantità di nutrienti da somministrare può essere facilmente regolata; e) i fluidi che accompagnano i nutrienti possono essere rimossi durante la stessa sessione di emodialisi. D'altra parte, devono essere riconosciuti alcuni potenziali svantaggi dell'IDPN: a) i nutrienti sono forniti soltanto durante la sessione di emodialisi con un'evidente limitazione della quantità somministrabile; b) i nutrienti sono infusi per via endovenosa in un periodo relativamente breve, e questo può non essere fisiologico; c) l'IDPN è più costosa se paragonata all'integrazione orale; e) viene aumentato il carico di lavoro degli infermieri.

Nessun grave effetto collaterale è stato dimostrato nel caso dell'IDPN (37). L'ipoglicemia, l'iperglicemia e l'ipertrigliceridemia possono essere facilmente evitate con il monitoraggio frequente degli esami del sangue; comunque, non si consiglia di iniziare l'IDPN in un paziente che presenti valori basali di trigliceridi $\geq 300 \mathrm{mg} / \mathrm{dL}$. Inoltre, gli effetti avversi più frequentemente identificati durante l'emodialisi come sintomi digestivi, ipotensione e crampi muscolari si verificano con un'incidenza simile a quella osservata nei pazienti che ricevono una supplementazione orale intradialitica (37). La sicurezza dell'IDPN è adeguata, con un basso tasso di complicanze nei pazienti con ESRD $(33,35-37)$.

\section{Conclusione}

I pazienti sottoposti a emodialisi cronica sono ad alto rischio per quanto riguarda lo sviluppo della PEW. La PEW è una condizione frequentemente osservata in questa popolazione e associata a un aumento della mortalità e del rischio di altre complicanze importanti. Sono disponibili diverse modalità di supporto nutrizionale per i pazienti con ESRD, e l'IDPN rappresenta una modalità sicura ed efficace nei casi in cui la 

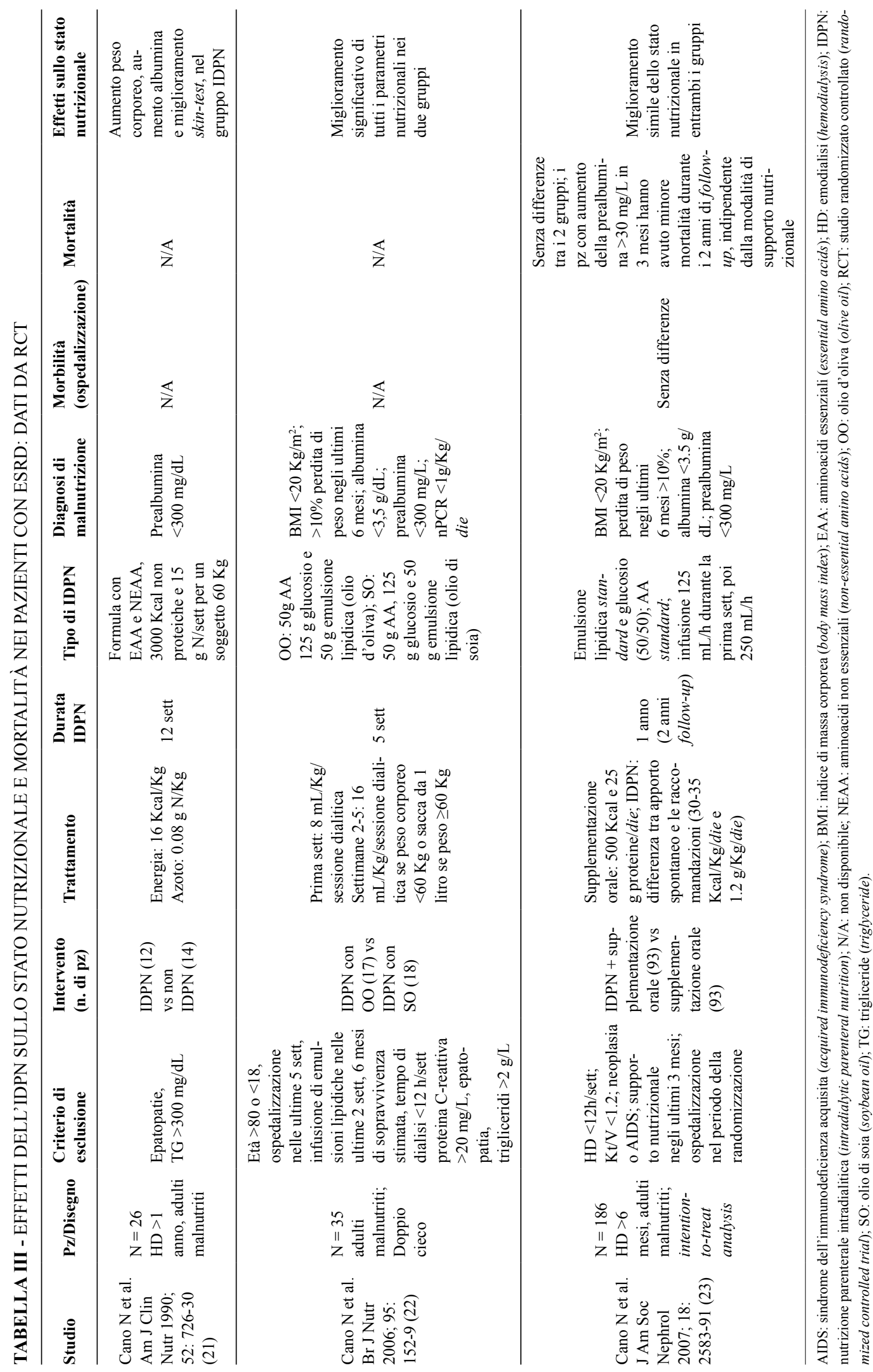
consulenza dietetica e la supplementazione orale siano inefficaci in caso di ridotta assunzione dietetica spontanea.

Sono necessari ulteriori studi per valutare definitivamente sia l'efficacia dell'IDPN a lungo termine in pazienti con ESRD in dialisi sia il rapporto costi-benefici.

\section{Riassunto}

La malnutrizione, o deplezione proteico-energetica (Protein Energy Wasting, PEW), è una condizione frequente nei pazienti con malattia renale cronica in stadio terminale (End Stage Renal Disease, ESRD) e si associa a un significativo aumento del rischio di mortalità e morbilità già caratteristicamente elevato in questa popolazione.

Dal momento che uno dei meccanismi principali della PEW nell'ESRD è rappresentato dall'insufficiente assunzione di nutrienti, la supplementazione nutrizionale orale viene ampiamente utilizzata come efficace prima scelta nella prevenzione e nel trattamento della PEW.

Tra gli altri approcci proposti come modalità di supporto nutrizionale per i pazienti con ESRD, la nutrizione parenterale intradialitica (IDPN) ha assunto di recente un ruolo importante in pazienti selezionati. Tuttavia, sebbene l'IDPN riesca a migliorare lo stato metabolico/nutrizionale nell'ESRD, le evidenze riguardo alla relazione tra questa modalità di supporto nutrizionale e una riduzione del tasso di ospedalizzazione e del rischio di mortalità sono ancora limitate.
Questa rassegna si propone di fare il punto sulle pratiche più utilizzate nel trattamento della PEW nell'ESRD, concentrandosi in particolare sul ruolo dell'IDPN come modalità di integrazione nutrizionale per i pazienti in emodialisi. A tale scopo, verranno illustrati gli aspetti quantitativi e qualitativi dell'IDPN, i problemi pratici legati alla sua gestione, le indicazioni e i limiti della metodica.

Parole chiave: Nutrizione parenterale intradialitica, Emodialisi cronica, Malnutrizione proteico-energetica, Supplementazione nutrizionale

Dichiarazione di conflitto di interessi: Gli Autori dichiarano di non avere conflitto di interessi.

Contributi economici agli Autori: Gli Autori dichiarano di non aver ricevuto sponsorizzazioni economiche per la preparazione dell'articolo.

\section{Indirizzo degli Autori:}

Prof. Enrico Fiaccadori

Dipartimento di Medicina Clinica e Sperimentale

Università degli Studi di Parma

Via Gramsci 14

43126 Parma

enrico.fiaccadori@unipr.it

\section{Bibliografia}

1. Fouque D, Kalantar-Zadeh K, Kopple J, et al. A proposed nomenclature and diagnostic criteria for protein-energy wasting in acute and chronic kidney disease. Kidney Int 2008; 73: 391-8.

2. Carrero JJ, Stenvinkel P, Cuppari L, et al. Etiology of the proteinenergy wasting syndrome in chronic kidney disease: a consensus statement from the International Society of Renal Nutrition and Metabolism (ISRNM). J Ren Nutr 2013; 23: 77-90.

3. Ikizler TA, Cano NJ, Franch H, et al. Prevention and treatment of protein energy wasting in chronic kidney disease patients: a consensus statement by the International Society of Renal Nutrition and Metabolism. Kidney Int 2013; in press.

4. U.S. Renal Data System. USRDS 2009 Annual Data Report: Atlas of Chronic Kidney Disease and End-Stage Renal Disease in the United States, National Institutes of Health, National Institute of Diabetes and Digestive and Kidney Diseases, Bethesda, MD 2009.

5. Kovesdy CP, Kalantar-Zadeh $\mathrm{K}$. Why is protein-energy wasting associated with mortality in chronic kidney disease? Semin Nephrol 2009; 29: 3-14.

6. Qureshi AR, Alvestrand A, Divino-Filho JC, et al. Inflamma- tion, malnutrition, and cardiac disease as predictors of mortality in hemodialysis patients. J Am SocNephrol 2002; 13 : S28-36.

7. Kalantar-Zadeh K, Rhee C, Sim JJ, Stenvinkel P, Anker SD, Kovesdy CP. Why cachexia kills: examining the causality of poor outcomes in wasting conditions. J Cachexia Sarcopenia Muscle 2013; 4: 89-94.

8. da Costa JA, Ikizler TA. Inflammation and insulin resistance as novel mechanisms of wasting in chronic dialysis patients. Semin Dial 2009; 22: 652-7.

9. Bossola M, Tazza L, Giungi S, Luciani G. Anorexia in hemodialysispatients: an update. Kidney Int 2006; 70: 417-22.

10. Fouque D, Laville M. Low protein diets for chronic kidney disease in non diabetic adults. Cochrane Database Syst Rev 2009; (3): CD001892.

11. Kovesdy CP, Kopple JD, Kalantar-Zadeh K. Management of protein-energy wasting in non-dialysis-dependent chronic kidney disease: reconciling low protein intake with nutritional therapy. Am J Clin Nutr 2013; 97: 1163-77.

12. Kalantar-Zadeh K, Cano NJ, Budde K, et al. Diets and enteral supplements for improving outcomes in chronic kidney disease. Nat Rev Nephrol 2011; 7: 369-84.

13. Lacson E Jr, Wang W, Zebrowski B, Wingard R, Hakim RM. 
Outcomes associated with intradialytic oral nutritional supplements in patients undergoing maintenance hemodialysis: a quality improvement report. Am J Kidney Dis 2012; 60: 591-600.

14. Cheu C, Pearson J, Dahlerus C, et al. Association between oral nutritional supplementation and clinical outcomes among patients with ESRD. Clin J Am Soc Nephrol 2013; 8: 100-7.

15. Cano N, Aparicio M, Brunori G, et al. ESPEN Guidelines on Parenteral Nutrition: Adult Renal Failure. Clin Nutr 2009; 28: 401-14.

16. Dukkipati R, Kalantar-Zadeh K, Kopple JD. Is there a role for intradialytic parenteral nutrition? A review of the evidence. Am J Kidney Dis 2010; 55: 352-64.

17. Kalantar-Zadeh K, Ikizler TA. Let them eat during dialysis: an overlooked opportunity to improve outcomes in maintenance hemodialysis patients. J Ren Nutr 2013, 3: 157-63.

18. National Kidney Foundation. K/DOQI clinical practice guidelines for nutrition in chronic renal failure. Am J Kidney Dis 2000; 35 (Suppl.): S1-S104.

19. Fouque D, Vennegoor M, ter Wee P, et al. EBPG guideline on nutrition. Nephrol Dial Transplant 2007; 22: ii45-87.

20. Fuhrman MP. Intradialytic parenteral nutrition and intraperitoneal nutrition. Nutr Clin Pract 2009; 24: 470-80.

21. Sezer S, Bal Z, Tutal E, Uyar ME, Acar NO. Long-Term Oral Nutrition Supplementation Improves Outcomes in Malnourished Patients With Chronic Kidney Disease on Hemodialysis. JPEN J Parenter Enteral Nutr 2014. Epub ahead of print.

22. Cockram DB, Hensley MK, Rodriguez MS et al. Safety and tolerance of medical nutritional products as sole sources of nutrition in people on hemodialysis. J Ren Nutr 1998; 8: 25-33.

23. Veeneman JM, Kingma HA, Boer TS, et al. Protein intake during hemodialysis maintains a positive whole body protein balance in chronic hemodialysis patients. Am J Physiol Endocrinol Metab 2003; 284: E954-65.

24. Pupim LB, Majchrzak KM, Flakoll PJ, Ikizler TA. Intradialytic oral nutrition improves protein homeostasis in chronic hemodialysis patients with deranged nutritional status. J Am Soc Nephrol 2006; 17: 3149-57.

25. Sundell MB, Cavanaugh KL, Wu P, et al. Oral protein supplementation alone improves anabolism in a dose-dependent manner in chronic hemodialysis patients. J Ren Nutr 2009; 19 : 412-21.

26. Rattanasompattikul M, Molnar MZ, Lee ML, et al. AntiInflammatory and Anti-Oxidative Nutrition in Hypoalbuminemic Dialysis Patients (AIONID) Study: results of the pilot-feasibility double-blind randomized placebo-controlled trial. J Cachexia Sarcopenia Muscle 2013; 4: 247-57.

27. Sayce HA, Rowe PA, McGonigle RJS. Percutaneous endoscopic gastrostomy feeding in haemodialysisout-patients. J Hum Nutr Dietet 2000; 13: 333-41.
28. Wolfson M, Jones MR, Kopple JD. Amino acid losses during hemodialysis with infusion of amino acids and glucose. Kidney Int 1982; 21: 500-6.

29. Capelli JP, Kushner H, Camiscioli TC, Chen S, Torres MA. Effect of intradialytic parenteral nutrition on mortality rates in endstage renal disease care. Am J Kidney Dis 1994; 23: 808-16.

30. Chertow GM, Ling J, Lew NL, et al. The association of intradialytic parenteral nutrition administration with survival in hemodialysis patients. Am J Kidney Dis 1994; 24: 912-20.

31. Hiroshige K, Iwamoto M, Kabashima N, Mutoh Y, Yuu K, Ohtani A. Prolonged use of intradialysis parenteral nutrition in elderly malnourished chronic hemodialysis patients. Nephrol Dial Transplant 1998; 13: 2081-7.

32. Cherry N, Shalansky K. Efficacy of intradialytic parenteral nutrition in malnourished hemodialysis patients. Am J HealthSyst Pharm 2002; 59: 1736-41.

33. Joannidis M, Rauchenzauner M, Leiner B, et al. Effect of intradialytic parenteral nutrition in patients with malnutritioninflammation complex syndrome on body weight, inflammation, serum lipids and adipocytokines: results from a pilot study. Eur J Clin Nutr 2008; 62: 789-95.

34. Dezfuli A, Scholl D, Lindenfeld SM, Kovesdy CP, KaantarZadeh K. Severity of hypoalbuminemia predicts response to intradialytic parenteral nutrition in hemodialysis patients. J Ren Nutr 2009; 19: 291-7.

35. Cano N, Labastie-Coeyrehourq J, Lacombe P, et al. Perdialytic parenteral nutrition with lipids and amino acids in malnourished hemodialysis patients. Am J Clin Nutr 1990; 52: 726-30.

36. Cano NJ, Saingra Y, Dupuy AM, et al. Intradialytic parenteral nutrition: comparison of olive oil versus soybean oil-based lipid emulsions. Br J Nutr 2006; 95: 152-9.

37. Cano N, Fouque D, Roth H, et al. Intradialytic parenteral nutrition does not improve survival in malnourished hemodialysis patients: a 2-year multicenter, prospective, randomized study. J Am Soc Nephrol 2007; 18: 2583-91.

38. Pupim LB, Flakoll PJ, Brouillette JR, et al. Intradialytic parenteral nutrition improves protein and energy homeostasis in chronic hemodialysis patients. J Clin Invest 2002; 110: 483-92.

39. Pupim LB, Flakoll PJ, Ikizler TA. Nutritional supplementation acutely increases albumin fractional synthetic rate in chronic hemodialysis patients. J Am Soc Nephrol 2004; 15: 1920-6.

40. Pupim LB, Flakoll PJ, Levenhagen DK, Ikizler TA. Exercise augments the acute anabolic effects of intradialytic parenteral nutrition in chronic hemodialysis patients. Am J Physiol Endocrinol Metab 2004; 286: E589-97.

41. Ikizler TA, Pupim LB, Brouillette JR, et al. Hemodialysis stimulates muscle and whole body protein loss and alters substrate oxidation. Am J Physiol Endocrinol Metab 2002; 282: E107-16. 